\title{
Éditorial : La condition de réfugié·e : expériences subjectives et mobilisations collectives
}

Editorial: The Condition of Refugee: Subjective Experiences and Collective Mobilizations

Editorial: Condición de refugiado: experiencias subjetivas y movilizaciones colectivas

Karen Akoka, Olivier Clochard et Albena Tcholakova

\section{(2) OpenEdition}

Journals

Édition électronique

URL : https://journals.openedition.org/remi/8915

DOI : 10.4000/remi.8915

ISSN : $1777-5418$

Traduction(s) :

Editorial: The Condition of Refugee: Subjective Experiences and Collective Mobilisations - URL :

https://journals.openedition.org/remi/14291 [en]

Éditeur

Université de Poitiers

Édition imprimée

Date de publication : 1 décembre 2017

Pagination : $7-21$

ISBN : 979-10-90426-60-3

ISSN : 0765-0752

Référence électronique

Karen Akoka, Olivier Clochard et Albena Tcholakova, «Éditorial : La condition de réfugiée : expériences subjectives et mobilisations collectives », Revue européenne des migrations internationales [En ligne], vol. 33 - n4 | 2017, mis en ligne le 01 décembre 2019, consulté le 14 avril 2022. URL : http:// journals.openedition.org/remi/8915; DOI : https://doi.org/10.4000/remi.8915 


\title{
Éditorial
}

\section{La condition de réfugié·e : expériences subjectives et mobilisations collectives}

\author{
Karen Akoka ${ }^{1}$, Olivier Clochard ${ }^{2}$ \\ et Albena Tcholakova ${ }^{3}$
}

Mercredi 8 janvier 2014, une tente entourée de banderoles sur lesquelles figurent différents slogans est installée devant l'entrée principale du ministère de l'Intérieur chypriote à Nicosie (cf. Photographie 1). Trois personnes reconnues réfugiées à Chypre depuis près de dix ans sont en grève de la faim depuis plusieurs semaines; elles demandent aux autorités de leur accorder des droits similaires à ceux des Chypriotes, d'agir contre les discriminations qu'elles subissent et pour le droit à la naturalisation. Quelques jours plus tard, un accord oral est conclu entre ces réfugiés, un responsable du HCR et un représentant du ministère de l'Intérieur, mais le temps passe et leur situation n'évolue pas. Six mois plus tard, ils reprennent leur grève de la faim et cessent à nouveau de $s^{\prime}$ hydrater ${ }^{4}$. Face au risque vital encouru au cœur de l'été chypriote par ces corps en résistance, les autorités finissent par consentir à leurs demandes.

L'idée du statut de réfugié comme aboutissement, voire comme terme, d'un long et douloureux processus est largement répandue. Or comme le montre l'exemple des réfugiés chypriotes relaté ci-dessus et certaines études scien-

\footnotetext{
1 Sociologue, maîtresse de conférences en science politique, Université Paris Nanterre, Institut des Sciences sociales du Politique (ISP), Maison Max Weber, 3ème étage, 200 avenue de la République, 92001 Nanterre Cedex; k.akoka@ parisnanterre.fr

2 Géographe, chargé de recherche au CNRS, Université de Poitiers, MIGRINTER, MSHS, Bâtiment A5, 5 rue Théodore Lefebvre, TSA 21103, 86073 Poitiers cedex 9 ;

olivier.clochard@univ-poitiers.fr

3 Sociologue, maîtresse de conférences, Université de Lorraine, IUT de Longwy, 186 rue de Lorraine, 54400 Cosnes-et-Romain ; albena.tcholakova@univ-lorraine.fr

4 Pour plus de précisions, voir la lettre datée du 10 juin 2014 et rédigée par les trois protagonistes, "Letter of recognized refugees to UNHCR Representative for Southern Europe ", [en ligne]. URL : https://kisa.org.cy/10-06-2014-letter-of-recognized-refugees-tounhcr-representative-for-southern-europe/
} 
tifiques, institutionnelles et associatives ${ }^{5}$, cette idée mérite d'être largement nuancée. Les difficultés traversées durant les mois, voire les années d'instruction des demandes d'asile sont-elles réellement de nature à cesser avec l'accès à un statut juridique même lorsqu'il apparaît fortement protecteur et générateur de droits ? Qu'est-ce que l'attention aux difficultés et aux ressources de ce temps délaissé de "l'après " peut nous enseigner sur la consistance du statut de réfugié ? Comment les différents usages et expériences sociales du statut peuvent-ils nous renseigner sur les dimensions à la fois individuelles et collectives de la condition de réfugié ?

Photographie 1 : Trois réfugiés reconnus en grève de la faim devant l'entrée principale du ministère de l'Intérieur chypriote

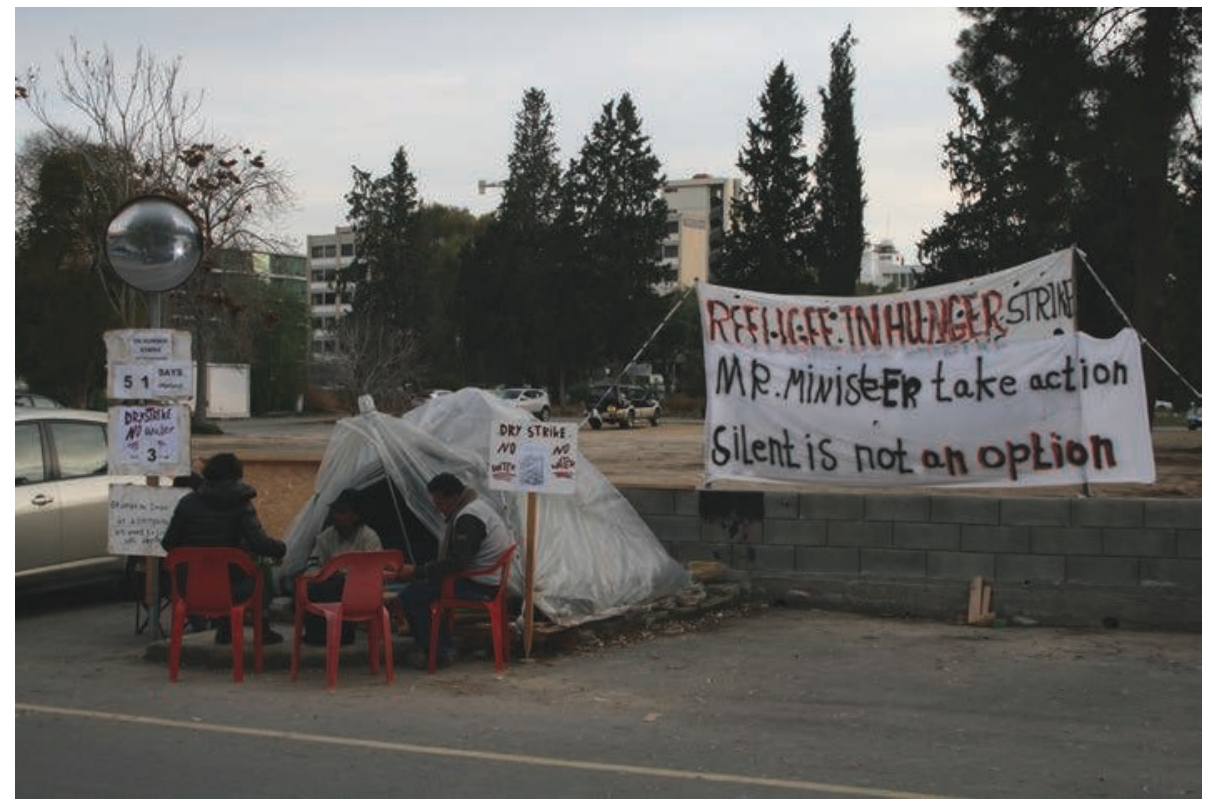

Crédit : 0. Clochard, Nicosie (Chypre), 8 janvier 2014.

Les différents articles réunis dans ce dossier répondent à ces questions chacun à leur manière, à partir de terrains, de méthodes et de disciplines différentes. Malgré la diversité de leurs approches, ils apportent des éclairages complémentaires à plusieurs questions communes qui renvoient à autant de facettes et de dimensions de la condition de réfugié : celles de l'expérience subjective du statut et des conditions individuelles et collectives de sa réappropriation ou de son rejet; celles des ressources qu'il permet de mobiliser qu'elles soient symboliques (reconnaissance) ou matérielles (travail) et de leurs

5 En France, sur les sites des associations Forum Réfugiés et France Terre d'Asile, sont présentés respectivement le programme d'intégration des réfugiés AccelAir et les activités de l'Observatoire de l'intégration des réfugiés. Des publications de I'UNHCR (2013a et $2013 b)$ portent sur l'insertion de ces populations dans les sociétés d'accueil des pays européens avec une attention particulière accordée aux mesures d'hébergement. Voir également le travail d'accompagnement des réfugiés (notamment dans leur projet de création d'entreprise) de l'association Singa France. 
limites ; celles des assignations vers lesquelles il ouvre et celles qu'il déplace ou reproduit.

Dans l'éditorial de ce dossier nous montrerons tout d'abord que les " réfugiés reconnus " restent des figures délaissées de la recherche au travers d'un état de l'art de la production scientifique sur les réfugiés. Nous rappellerons ensuite les enjeux démographiques et juridiques dans lesquels les catégories administratives de "réfugié reconnu " s'inscrivent à l'échelle européenne. Nous présenterons enfin les articles de ce dossier et les principales problématiques qui se dégagent de leur mise en commun.

\section{Les "réfugiés reconnus ": figures délaissées de la recherche}

À partir de ce qui a été communément appelé " la crise de l'asile " des années 1990 en Europe, les questionnements autour du sujet atteignent une large visibilité dont témoigne la mise en place de politiques restrictives, de plus en plus subordonnées aux impératifs de maîtrise des flux migratoires. À la même période se développent également de nouveaux champs de recherche scientifique sur la question, en particulier les refugee studies et les forced migration studies. Ces deux sous-champs pluridisciplinaires, réunissant des juristes, des sociologues, des anthropologues, des politistes, des économistes, des géographes, et donnant lieu à des débats en Grande Bretagne, au Danemark, au Canada ou aux États-Unis ${ }^{6}$ (Hathaway, 2007 ; Malkki, 1995 ; Lassailly-Jacob, 1999 ; Martin, 1982 ; Turton, 2003 ; Castles, 2003a ; Fiddian-Qasmiyeh et al., 2014) se sont longtemps développés de manière cloisonnée par rapport au champ des migrations internationales. Depuis quelques années, ils se sont néanmoins rapprochés pour donner lieu à une production féconde qui permet d'explorer de nouveaux sujets comme les expériences individuelles, familiales et collectives de l'enfermement, de l'encampement, du déplacement, du retour, de la dépossession et de "l'intégration " des demandeurs d'asile, des déplacés, des réfugiés et ce, dans différents contextes géographiques (Fiddian-Qasmiyeh, 2014 ; Bakewell, 2008 ; Yi Cheung et Phillimore, 2013 ; Van Hear, 2014). L'essentiel des analyses développées dans les refugee/forced migrations studies porte cependant sur les procédures nationales et internationales de détermination du statut de réfugié, de protection des demandeurs d'asile et des réfugiés (Martin, 1982 ; Boyd, 1999 ; Goodwin-Gill, 2014), sur la construction des politiques publiques (Ferris, 1985 ; Castles, 2003b), sur le rôle des institutions internationales, étatiques et non gouvernementales dans la protection et l'assistance aux réfugiés (Harrell-Bond, 1986) et sur la " gestion des indésirables" (Agier, 2008), sur la " raison humanitaire " et le " humanitarian business " (Fassin, 2010 ; Weiss, 2013), ou encore sur la fabrique des catégories de l'action publique (Turton, 1996 ; Bakewell, 2008).

Plus spécifiquement, dans les pays européens, qui font l'objet des articles du présent dossier, les travaux académiques sur l'asile se centrent sur la genèse, les causes et les dynamiques des situations d'exil (Bolzman, 1996 ; Doraï, 2006 ;

6 Le Refugee Studies Centre, au sein de I'Université d'Oxford, joue un rôle de figure de proue. Voir aussi les travaux développés au sein du York University in Toronto et du Refugee Policy Group in Washington DC, pour ne noter ici que ces quelques espaces académiques. 
Vahabi, 2008 ; Vinatier, 2009) ; les processus historiques d'institutionnalisation du dispositif d'asile (Akoka, 2012, Akoka et Spire, 2013 ; Angoustures et al., 2017 ; Masse, 2001 ; Kobelinsky, 2010) et les politiques contemporaines en matière d'asile appréhendées comme une production "d'indésirables " (Agier, 2002, 2008 et 2011 ; Clochard, 2007 ; Valluy, 2009 ; Migreurop, 2017). La précarisation continue du statut de demandeur d'asile et la question de l'usure des travailleurs sociaux ont conduit quant à elles à porter le regard sur celles et ceux qui accompagnent les demandeurs d'asile (Colin et Laval, 2005). Des travaux croisant des approches juridiques et socio-anthropologiques étudient la quête $d^{\prime}$ asile et la construction sociale et juridique de l'aide à l'accès aux droits des exilés en s'intéressant de plus près aux expériences et aux pratiques des requérants et des travailleurs sociaux (Franguiadakis, Jaillardon et Belkis, 2004 ; Halluin-Mabillot, 2012). Parallèlement une approche psychologique et psychiatrique de la santé mentale des demandeurs d'asile se développe (Bruyère, 2004 ; Grinberg et Grinberg, 1987 ; Pestre, 2010 ; Saglio-Yatzimirsky, 2018). En résumé les dispositifs de droit, les dispositifs de contrôle, de mise à l'écart et de gestion de l'attente retiennent l'attention des chercheurs qui partent souvent de la nécessité de rendre visibles ceux que ces dispositifs tendent à invisibiliser. Ces différentes perspectives de recherche ont fait la preuve de leur fécondité, mais elles n'ont jamais porté centralement sur la question du devenir social des réfugiés, une fois le statut obtenu.

Parmi les travaux, plus rares, s'intéressant directement aux réfugiés reconnus, les perspectives macroscopiques tendent à dominer au travers d'études sur I'accès à l'emploi, au logement et à la santé des réfugiés dans plusieurs états européens, en particulier la Grande-Bretagne et la Suède (Korac, 2003 ; Bloch, 2007 ; Valtonen, 2001 ; Atfield et al., 2007 ; Craig et al., 2006 ; Chang, 2008 ; Åslund, 2002 ; Åslund et Fredrikksson, 2005 ; Kogan, 2003 ; Lundborg, 2010 ; Bevelander et Pandakur, 2012 ; Rea et Wets, 2015). Un certain nombre de recherches qualitatives sur les réfugiés reconnus ont néanmoins commencé à se développer cette dernière décennie, généralement centrées sur des thématiques bien spécifiques telles la politisation et les mobilisations politiques des réfugiés (Dufoix, 2000 ; Étiemble, 2004) ; la santé mentale des réfugiés (Barou et Blanco, 2011) ou l'accès des réfugiés statutaires au logement (Ducheny, 2008). Mais c'est sans conteste la question du travail qui occupe la part la plus importante de la petite production sur les réfugiés reconnus, depuis l'étude socio-historique de Spire (2004) sur les politiques de mise au travail des réfugiés pour la période 1945-1975, les recherches de Billion (2001) et de Meslin (2011) sur les parcours professionnels des réfugiés originaires d'Asie du Sud-Est (1970-1980) dont certains sont devenus des "travailleurs ouvriérisés ", en passant par l'étude croisée sur la quête de travail des réfugiés en France et en Bulgarie de Tcholakova (2012).

\section{Réfugiés en Europe : une géographie contrastée des politiques d'accueil et d'intégration}

Les articles de ce dossier sont circonscrits à l'espace européen, marqué dès I'entre-deux-guerres par d'importants mouvements de personnes devenues apatrides dans le contexte de l'éclatement des grands empires, de l'instauration de nouveaux États-nations et des dénationalisations massives. Face à ces situations et dans le cadre de la Société des Nations (SDN), ce sont d'abord des dispositifs spécifiques selon les nationalités qui sont mis en place par les 
grands États européens, comme le rappelle I'article d'Anouche Kunth pour les Arméniens. Après la Seconde Guerre mondiale, c'est dans le cadre des Nations unies que dix-neuf États ${ }^{7}$ adoptent un système de protection pour les Européens fondé sur une conception individualiste de la notion de réfugié, la convention de Genève du 28 juillet 1951. Seize ans plus tard, le 31 janvier 1967, dans le contexte d'une guerre froide qui a étendu ses luttes idéologiques aux pays dits du Sud, le protocole additionnel de NewYork, ouvre le champ d'application de la convention de Genève aux événements survenus après le $1^{\text {er }}$ juillet 1951 et aux ressortissants de l'ensemble des États des autres continents.

Près de soixante-dix ans après l'adoption du protocole additionnel de New York, et contrairement aux idées reçues, l'Europe n'accueille qu'une petite partie des réfugiés dans le monde. Selon I'UNHCR, I'Union européenne comptait à la fin de l'année 2016 près de 2 millions de personnes réfugiées (soit 0,4\% de la population européenne) $)^{8}$. Ces chiffres seraient largement supérieurs si les gouvernements européens appliquaient de façon moins restrictive d'une part les procédures relatives à l'octroi du statut de réfugié, et d'autre part celles liées au rapprochement familial des réfugiés. Dans une note publiée en 2014, JeanMichel Belorgey, juge à la Cour Nationale du Droit d'Asile (CNDA) montre que le rapprochement familial est en France dans l'impasse, notamment parce que " des consulats français se livrent à des vérifications d'état civil qui n'ont pas lieu d'être ou ne devraient pas pouvoir être faites auprès du pays de persécution ${ }^{9}$. Ces logiques atteignent des dimensions paroxystiques dans certains pays européens, comme à Chypre, où les réfugiés patientent parfois plus de dix ans, avant de pouvoir bénéficier du rapprochement familial, tel cet Ivoirien, rencontré en 2013 , et en attente depuis 2005 de l'autorisation de faire venir ses enfants ${ }^{10}$.

De part et d'autre de la moyenne globale du taux de reconnaissance en Europe $(27,1 \% \text { en } 2008 ; 31,5 \% \text { en } 2013 ; 45,5 \% \text { en } 2017)^{11}$, la disparité des taux nationaux dessine ainsi une géographie des politiques européennes d'accès au statut, que la diversité des droits et ressources auxquelles il donne lieu vient compléter, invitant à s'interroger sur la pertinence même de l'appellation commune de " réfugié " dans les différents pays du continent. Bien qu'en vertu des directives européennes, toute personne reconnue "réfugiée " doit pouvoir bénéficier d'un " ensemble de droits uniformes aussi proches que possible de

7 Allemagne, Autriche, Belgique, Brésil, Colombie, Danemark, France, Grèce, Israël, Italie, Liechtenstein, Luxembourg, Norvège, Pays-Bas, Royaume-Uni, Vatican, Suède, Suisse, Turquie.

8 Pour rappel, selon I'UNHCR, le nombre de personnes déracinées à travers le monde du fait de conflits et de persécutions s'élevait à 65,6 millions à la fin de l'année 2016. On estime le nombre de réfugiés à près de 22,5 millions (à savoir des personnes ayant franchi une frontière internationale) et le nombre de personnes déplacées à près de 40,3 millions (soit des personnes ayant trouvé refuge à l'intérieur de leur pays). À ces derniers s'ajoutent les réfugiés palestiniens qui relèvent du mandat de I'UNWRA et dont le nombre est estimé à près de 5,3 millions.

9 Belorgey Jean-Michel, Le droit d'asile, bouc émissaire des anti immigrationnistes, 28 avril 2014, [en ligne]. URL : http://ecritures-et-societe.over-blog.com/article-le-droit-d-asilebouc-emissaire-des-anti-immigrationnistes-123462508.html

10 Notes de terrain (janvier 2014) issues d'entretiens réalisés à Chypre par Karen Akoka et Olivier Clochard.

11 Décisions prises en première instance (source : base de données Eurostat consultée en juin 2018). 
ceux dont jouissent les citoyens de l'Union ${ }^{12}$ et bien que la convention de 1951 rappelle elle-même que sur le plan de l'enseignement, de l'assistance, de la sécurité sociale, du travail, " les États accorderont aux réfugiés le même traitement qu'aux nationaux ", l'accès à ces droits continue de faire l'objet de tensions et reste très disparate au sein des divers pays européens.

En Italie où les taux annuels de reconnaissance (statut de réfugié, protection subsidiaire et statut humanitaire) sont parfois supérieurs à la moyenne européenne $(48,1 \%$ de décisions positives en première instance en $2008,60,1 \%$ en $2013,40,6 \%$ en 2017) ${ }^{13} c^{\prime}$ est surtout, pour reprendre les termes d'un porteparole du HCR, " ce qui vient après qui n'est pas satisfaisant ", telles l'absence de revenus fixes ou l'incapacité de trouver un emploi stable ${ }^{14}$. Nils Muiznieks, commissaire aux droits de l'homme, souligne quant à lui « la quasi-absence d'un cadre d'intégration ${ }^{15}$ pour les réfugiés en Italie. Des constats que le rapport de la CFDA (2008: 100) avait déjà soulignés en 2008 en pointant le nombre important d'Érythréens ayant obtenu une protection subsidiaire en Italie, pourtant présents dans les jungles du Calaisis, dans I'attente de gagner la Grande-Bretagne.

La durée du titre de séjour octroyé aux réfugiés statutaires est, quant à elle, également très différente d'un pays européen à l'autre. Les bénéficiaires de la convention de Genève ont droit à une carte de résident de dix ans en France et à une carte de séjour de cinq ans en Espagne ou en Italie ${ }^{16}$ (renouvelable de plein droit dans les deux cas). À Chypre, cette durée tombe à trois ans, de même qu'en Roumanie où les réfugiés reconnus doivent prouver qu'ils résident dans le pays à l'aide d'un titre de propriété ou d'un contrat de location afin d'obtenir leur carte de séjour ${ }^{17}$.

Le droit à l'installation des réfugiés dans un autre pays que celui dans lequel ils ont bénéficié d'une protection est également variable selon les pays. Si I'Union européenne a modifié en 2011 la directive relative au statut de résident " longue durée " de 2003 pour qu'il puisse être accordé aux réfugiés statutaires, d'importantes différences demeurent. Un réfugié reconnu en France peut par exemple s'installer en Belgique ce qui n'est pas le cas pour un réfugié reconnu à Chypre ou en Bulgarie. Le titre de séjour de trois ans délivré aux réfugiés par les autorités chypriotes ou bulgares n'étant pas considéré par I'Union européenne comme un titre de résident "longue durée " ses bénéficiaires ne peuvent que circuler, mais pas s'installer en Europe.

12 Parlement européen, Conseil européen de Tampere, 15 et 16 octobre 1999, [en ligne]. URL : http://www.europarl.europa.eu/summits/tam_fr.htm

13 Chiffres extraits en mai 2018 de la base de données d'Eurostat.

14 Laura Boldrini, porte-parole du HCR en Italie, dans un article du New York Times du 21 décembre 2012 d'Elisabetta Povoledo, "The Italian Paradoxe on Refugees ".

15 Commissaire aux droits de I'homme, 18 septembre 2012, "L'Italie doit accélérer les procédures judiciaires et améliorer le traitement des Roms et des migrants ".

16 Source : entretien avec Alessandra Capodanno, Migreurop, le 11 février 2014.

17 Le site de l'inspectorat général pour l'immigration indique que les étrangers ayant obtenu une forme de protection de l'État roumain peuvent obtenir un permis de séjour pour prouver leur identité et sortir du pays. Pour en faire la demande, ils doivent apporter la preuve que leur résidence est en Roumanie (titre de propriété, contrat de location enregistré à l'administration fiscale, certificat d'hébergement authentifié ou tout document valable auprès de I'administration roumaine qui prouve le versement d'une allocation). 
Depuis le traité d'Amsterdam (1999), les États membres n'ont cessé de rappeler leur volonté d'instituer un régime d'asile européen commun qui consisterait notamment " à mettre en place un statut de réfugié uniforme valable dans toute I'Union ", promesse réaffirmée en décembre 2009, au moment de l'adoption du programme de Stockholm où le Conseil européen avait souligné " son attachement [...] à établir, d'ici à 2012, un espace commun de protection et de solidarité fondé sur une procédure d'asile commune et un statut uniforme pour les personnes bénéficiant d'une protection internationale ". La politique européenne d'asile continue pourtant d'être marquée par la juxtaposition de dispositifs nationaux bien plus que par l'harmonisation malgré l'adoption de directives qui rappellent régulièrement qu'une " telle politique devrait être régie par le principe de solidarité, y compris sur le plan financier, entre les États membres ». Derrière cette litanie de bonnes intentions force est de constater que la politique d'asile européenne est non seulement passée de la protection des réfugiés à la protection contre l'arrivée des demandeurs d'asile, pour reprendre la formule de Bouteillet-Paquet (2001), mais que la précarisation continue du statut dessine des expériences sociales disparates de la condition de réfugié.

\section{La condition de réfugié : usages et expériences après la reconnaissance}

Ce dossier se propose de porter le regard sur la période qui suit l'obtention du statut de réfugié, souvent considéré comme un aboutissement. S'il est vrai que cette obtention représente, à l'échelle subjective des individus, un tournant éclairant les processus de catégorisations et d'assignations bureaucratiques, juridiques et administratives, le moment $d^{\prime}$ " après " ne constitue pas moins un sujet intéressant. $C^{\prime}$ est ce que montrent chacun à leur manière les différents articles de ce dossier.

Anouche Kunth se centre sur la période de l'entre-deux-guerres en s'intéressant au rapport des premiers réfugiés à leur statut et aux administrations qui le gèrent. Elle examine les réalités concrètes que recouvre ce statut marqué par une indétermination pratique et juridique. Au travers de cette approche, l'auteure retrace la manière dont les réfugiés eux-mêmes, et en particulier les réfugiés arméniens, ont compris la portée de cette catégorie administrative, une catégorie qu'ils se réapproprient pour la transformer en groupe d'appartenance. L'entrée par la catégorie est ainsi le moyen pour l'auteure de saisir les relations entre des sujets et des dispositifs du pouvoir, mais aussi d'appréhender des subjectivités dans leur rapport personnel à un statut qu'ils rejettent ou revendiquent.

Karine Meslin analyse quant à elle les effets sociaux de la désignation comme réfugié à partir d'une recherche sur les Cambodgiens reconnus réfugiés dans les années 1980 et installés dans la région des Pays de la Loire. La longue durée de leur présence en France (trente ans) permet à l'auteure de livrer une analyse longitudinale qui intègre les secondes générations. Elle montre les efforts persistants des ainés pour coller aux représentations sur les réfugiés en général (vus comme des étrangers aux fortes ressources socio-culturelles) et sur les réfugiés cambodgiens en particulier (vus comme des étrangers dociles et intégrés). Cet effort passe par un travail de mise à distance de la figure sociale de l'immigré et des préjugés négatifs qui en découlent. II se déploie ainsi par un recours aux catégories administratives et aux stéréotypes ethnicisant. 
Barbara Herman et Andréa Rea proposent une étude statistique de l'intégration sur le marché du travail des réfugiés en Belgique à partir de données administratives longitudinales et du concept de "carrière migratoire ". Ils montrent que le temps constitue le facteur principal pour sortir de la dépendance à l'aide sociale, entrer sur le marché du travail et changer de position socioéconomique. Au-delà du facteur temporel, les auteurs mettent en valeur trois autres grands déterminants de l'insertion socioéconomique des réfugiés : les conditions structurelles (institutionnelles et politiques), les caractéristiques individuelles (âge, genre, situation familiale et nationalité) et la mobilisation des réseaux sociaux. Contrairement aux stéréotypes, ils montrent que les réfugiés subsahariens ont les plus grandes probabilités d'avoir une "carrière active " en Belgique, émettant I'hypothèse de la langue française (parlée par un nombre important d'entre eux) comme facteur clef de cette réussite. Ils montrent enfin les effets relatifs d'être reconnu réfugié en termes socioéconomiques puisque l'obtention du statut vient plutôt consolider la situation de personnes déjà bien insérées sur le marché du travail avant d'être reconnues réfugiées. Dans la même veine, I'acquisition du statut conduit à un changement important de secteurs d'activité (glissement du secteur de l'agriculture vers les secteurs de l'industrie, de l'administration publique, de la santé, du social, de l'hébergement, de la restauration et du commerce) pour ceux qui occupaient déjà un emploi. Sous l'angle de l'insertion sur le marché du travail, il apparaît ainsi que la reconnaissance du statut de réfugié vient davantage renforcer une position antérieure que faire basculer des sujets dans une nouvelle condition.

Angelina Étiemble propose, quant à elle, une réflexion sur les processus de politisation des réfugiés tamouls, première population de réfugiés en France depuis la fin des années 1990, et sur le statut de réfugié comme vecteur de mobilisation collective. Son article montre, à partir d'une enquête ethnographique du quartier de la Chapelle à Paris, que la politisation opère dans l'ombre du mouvement tamoul de libération nationale (LTTE), dit des "Tigres ", qui investit le statut de réfugié comme ressource pour la reconnaissance de sa cause. La vie quotidienne des réfugiés tamouls, mais aussi leurs projets et le sens donné à leur exil sont ainsi encadrés par les militants tamouls. Ces derniers promeuvent une " historiographie officielle " et une interprétation nationaliste de l'exil qui se doit d'être entièrement consacrée au soutien de la " cause tamoule ". Dans ce contexte, le terme " réfugié " ou " exilé " qui confère une reconnaissance au combat des tamouls au Sri Lanka est valorisé au détriment de celui de " diaspora "; les projets d'immigration vers la Grande-Bretagne sont désapprouvés en tant qu'ancienne puissance coloniale hostile à la cause tamoule ; et les naturalisations sont encouragées en tant que moyens de légitimer la cause sur la scène française. Ce cadrage identitaire, la promotion d'un mode de vie tamoul en exil et l'organisation d'une vie communautaire orientée vers le sacrifice pour le projet collectif du " peuple tamoul ", s'opère largement via les associations du quartier de la Chapelle qui mêlent dimensions politiques, humanitaires et culturelles afin d'assurer l'adhésion des Tamouls à leur cause. Avec l'interdiction des LTTE en Europe (2006) et leur défaite au Sri Lanka (2009), cette emprise s'affaiblit ; les activités associatives et religieuses se tournent davantage vers des liens culturels et linguistiques que vers le seul projet politique ; le terme " diaspora " est revalorisé ainsi qu'une ethnicité tamoule sans substrat nationaliste. 
Albena Tcholakova s'intéresse à la condition de réfugié en Bulgarie, pays spécifique par rapport à la plupart des grands États européens et méditerranéens, en tant que terre d'émigration et d'immigration récente. Elle interroge l'expérience du statut de réfugié dans ce contexte particulier à partir du prisme du travail. Cette entrée lui permet d'ouvrir le regard sur l'expérience sociale et symbolique du statut de réfugié en mettant au jour les distorsions entre dureté du contexte bulgare du point de vue de l'accès aux droits et au travail d'un côté et les attentes d'accès à une citoyenneté sociale et politique des réfugiés de l'autre. Le travail en tant qu'espace social, institutionnel et économique y est exploré comme expression et activation d'une domination protéiforme (genre, classe, race, etc.), mais aussi comme espace de réhabilitation de soi, pour soi et auprès des autres. L'auteure montre les rapports sociaux de domination à I'œuvre et la manière dont le travail participe du renforcement des normes de genre et d'ethnicisation qui pèsent sur les réfugiés et auxquelles leur nouveau statut leur permet difficilement d'échapper.

\section{Des identités subjectives aux mobilisations collectives}

À partir de terrains (France, Bulgarie, Belgique), de groupes (Arméniens, Africains, Cambodgiens, Tamouls), de méthodes (enquête historique, ethnographique, quantitative) et en prenant appui sur des périodes et des temporalités très différentes, ces travaux trouvent plusieurs grands points de convergences qui fonctionnent comme autant d'éclairages et de pistes de réflexions sur la condition et l'expérience sociale de réfugié.

L'attention au temps de "l'après " permet d'abord de prolonger, en intégrant une étape supplémentaire, le travail de déconstruction de la qualité de réfugié, dans le sillage d'une longue lignée de travaux sur le statut (Harrell Bond ; 1986, Malkki, 1995 ; Noiriel, 1998 ; Halluin-Mabillot, 2012) entendu avant tout comme une construction sociale, mais aussi comme le produit de considérations (politiques, diplomatiques et socio-économiques) qui en disent plus long sur les sociétés qui labélisent, que sur ceux qui sont désignés comme réfugiés (Akoka, 2012). L'attention à "l'après " montre que le statut de réfugié peut s'appréhender certes comme un " produit ", mais aussi du point de vue de ses effets. Sous cet angle ce sont alors la diversité des droits et l'hétérogénéité de la condition de réfugié qui participent du travail de déconstruction du statut et $s^{\prime}$ avèrent riches en enseignements sur les sociétés qui le (re)configurent. $C^{\prime}$ est en ce sens que le choix de scruter "l'après " de la détermination du statut de réfugié dans des configurations nationales, politiques, sociales et historiques très diverses s'est avéré heuristique. Il a permis de donner chair à l'hétérogénéité des droits, des effets, des conditions et des expériences mis au jour entre des réfugiés arméniens, cambodgiens ou tamouls reconnus en France respectivement dans I'entre-deux-guerres, la fin des années 1970 et les années 1990-2000; ou des réfugiés reconnus dans la décennie 2010 en Belgique ou en Bulgarie.

L'analyse de "l'après " ouvre également le regard sur un temps plus long, et d'une certaine manière plus existentiel, que celui de la préparation et l'attente d'un verdict venu de l'extérieur. Une fois le statut de réfugié obtenu il s'agit de faire sienne (ou non) cette nouvelle identité juridico-administrative. Comment 
les personnes concernées répondent-elles à ces classifications administratives ? Comment s'emparent-elles des " identités de papiers " qui leur sont assignées ? Comment les investissent-elles ou les mettent-elles à distance et à quelles conditions ? Tenir ensemble la question de l'expérience avec celle de l'assignation, permet de rompre avec une division du travail sociologique quelque peu dichotomique entre, d'un côté, l'analyse des catégories sociales comme des identités imposées de l'extérieur et, de l'autre, celle des groupes sociaux comme l'aboutissement d'un travail de construction par et pour ses membres (Boltanski, 1982 ; Noiriel, 1997 ; Tcholakova, 2016).

Sans surprise la question de l'" après " ouvre sur la question du travail dans un nombre non négligeable des articles ici réunis. Karine Meslin montre que les préjugés positifs dont bénéficient les réfugiés cambodgiens avant même d'être en contact avec leurs employeurs ou collègues ont facilité depuis les années 1980 leur insertion professionnelle sans pour autant éviter leur déclassement social, la grande majorité d'entre eux étant ouvriers spécialisés ou employés. Barbara Herman et Andréa Rea mettent en exergue certains facteurs clefs de I'intégration des réfugiés au marché du travail en Belgique aujourd'hui et en premier lieu la durée de la présence. Albena Tcholakova montre les ambivalences de l'expérience du travail chez les réfugiés en Bulgarie, source de revenu, d'émancipation, espace de construction d'une sociabilité et d'une image de soi positive d'un côté et de l'autre, lieu de stigmate réduisant doublement les réfugiés à leur corps d'ouvrier (simple force de travail) et à leurs corps racisé. Anouche Kunth montre qu'à défaut d'une catégorie de réfugié comportant comme aujourd'hui un ensemble de droits stables et consolidés c'est en tant qu'ouvriers (des usines Schneider) que les Arméniens du Creusot qui ne savent pas encore qu'ils ont droit au statut de réfugié ont accès à des aides.

Le statut de réfugié apparaît enfin, dans un certain nombre des articles de ce dossier comme le support de différentes formes de mobilisations collectives. Karine Meslin montre I'usage par les réfugiés cambodgiens du statut de réfugié comme signe collectif de distinction permettant de différencier le groupe tout entier de la figure sociale de l'immigré et des stéréotypes négatifs qui lui sont associés. Ce travail de distinction se fait néanmoins au prix d'une violence symbolique exercée en particulier sur les fractions les plus populaires et les secondes générations, plus résistantes à l'injonction collective à I'hypercorrection. Dans le cas de réfugiés arméniens de l'entre-deux-guerres en France, étudiés par Anouche Kunth, la dimension collective est d'autant plus importante que le statut de réfugié n'a pas encore une dimension individuelle et que c'est au titre de l'appartenance au groupe national (ici celui des arméniens) que le statut de réfugié est accordé. Angélina Étiemble montre quant à elle que le statut de réfugié est investi par les militants et sympathisants des mouvements séparatistes tamouls en France comme le vecteur d'une forte reconnaissance pour leur cause. Dès lors, un puissant travail de cadrage et une forte pression sont exercés sur les membres du groupe pour promouvoir l'idée d'une appartenance collective à la communauté des réfugiés tamouls centrée sur l'attachement indéfectible au pays et à la cause, par opposition à une identification à la diaspora tamoule valorisant davantage les liens de solidarité au sein de la communauté diasporique. 


\section{Références bibliographiques}

Agier Michel (2011) Le Couloir des exilés. Être étranger dans un monde commun, Bellecombe-en-Bauge, Éditions du Croquant, 120 p.

Agier Michel (2008) Gérer les indésirables. Des camps de réfugiés au gouvernement humanitaire, Paris, Flammarion, $350 \mathrm{p}$.

Agier Michel (2002) Aux abords du monde, les réfugiés, Paris, Payot \& Rivages, $155 \mathrm{p}$.

Akoka Karen (2012) La fabrique du réfugié à I'OFPRA (1952-1992) : du consulat des réfugiés à l'administration des demandeurs d'asile, Thèse de doctorat en Sciences Sociales, Université de Poitiers, 519 p.

Akoka Karen et Spire Alexis (2013) Pour une histoire sociale de l'asile politique en France, Pouvoirs, Revue française d'études constitutionnelles et politiques, 144, pp. 67-77.

Angoustures Aline, Kevonian Dzovinar et Mouradian Claire (2017) Réfugiés et apatrides, administrer l'asile en France (1920-1960), Presses Universitaires de Rennes, $312 \mathrm{p}$.

Åslund Olof (2002) Integration of refugees in the Swedish labor market - policies and neighborhoods, Working Paper, Uppsala, Instute for Labour Market Policy Evaluation.

Åslund Olof and Fredrikksson Peter (2005) Ethnic enclaves and welfare cultures quasi experimental evidence, Working paper, 8, Uppsala, Instute for Labour Market Policy Evaluation.

Atfield Gaby, Brahmbhatt Kavita and O'ToolTherese (2007) Refugees' Experiences of Integration, Birmingham, Refugee Council and University of Birmingham, $76 \mathrm{p}$.

Bakewell Oliver (2008) Research beyond the Categories: The Importance of Policy Irrelevant Research into Forced Migration, Journal of Refugee Studies, 4 (21), pp. 432-453.

Barou Jacques et Blanco Domitille (2011) Parcours de réfugiés : le statut, et après ? Étude anthropologique menée auprès de réfugiés de l'agglomération lyonnaise, Réseau Samdarra.

Bevelander Pieter (2011) The employment integration of resettled refugees, asylum claimants, and family reunion migrants in Sweden, Refugee Survey Quarterly, 30 (1), pp. 22-43.

Bevelander Pieter and Pendakur Ravi (2012) The Labour Market Integration of Refugee and Family Reunion Immigrants: A Comparison of Outcomes in Canada and Sweden, IZA Discussion Paper, 6924, 27 p.

Billion Pierre (2001) Où sont passés les "travailleurs réfugiés " ? Trajectoires professionnelles des populations du Sud-Est asiatique, Hommes et Migrations, 1234 , pp. 38-49.

Bloch Alice (2007) Refugees in the UK labour market: The conflict between economic integration and policy-led labour market restriction, Journal of Social Policy, 37 (1), pp. 21-36.

Boltanski Luc (1982) Les cadres. La formation d'un groupe social, Éditions de Minuit, $528 \mathrm{p}$. 
Bolzman Claudio (2001) Politiques d'asile et trajectoires sociales des réfugiés : une exclusion programmée. Le cas de la Suisse, Sociologie et Sociétés, 33 (2), pp. 133-158.

Bolzman Claudio (1996) Sociologie de l'exil : une approche dynamique. L'exemple des réfugiés chiliens en Suisse, Zürich, Seismo, 333 p.

Bouteillet-Paquet Daphné (2001) L'Europe et le droit d'asile, Paris/Montréal/ Budapest/Turin, L'Harmattan, 396 p.

Boyd Monica (1999) Gender, Refugee Status and Permanent Settlement, Gender Issues, 1 (17), pp. 5-25.

Bruyère Blandine (2004) Le demandeur d'asile : objet du tortionnaire, sujet du travail psychothérapique, Le journal des psychologues, 217, pp. 41-43.

Castles Stephen (2003a) Toward a Sociology of Forced Migration and Social Transformation, Sociology, 77 (1), pp. 13-34.

Castles Stephen (2003b) The International Politics of Forced Migration, Socialist Register, 39, pp. 172-192.

Chang Jaclyn (2008) Beyond 365 Days. The Economic Integration of African Refugees in Vancouver, Canada, Dissertation in fulfilment of the requirements for the degree Masters in Forced Migration Studies, Faculty of Arts, University of the Witwatersrand, $111 \mathrm{p}$.

Clochard Olivier (2007) Le jeu des frontières dans l'accès au statut de réfugié. Une géographie des politiques d'asile et d'immigration, Thèse de doctorat en géographie, Université de Poitiers, 486 p.

Colin Valérie et Laval Christian (2005) Santé mentale et demandeurs d'asile en Région Rhône-Alpes. Modalités pratiques et inter partenariales, Rapport final, Recherche-action commanditée par la DRASS Rhône-Alpes, ORSPERE-ONSMP, $178 \mathrm{p}$

Coordination Française pour le Droit d'Asile (CFDA) (2008) La loi des jungles. La situation des exilés sur le littoral de la Manche et la Mer du Nord, Rapport coordonné par Karen Akoka et Olivier Clochard, 156 p., [en ligne]. URL : http:// cfda.rezo.net/download/La\%20loi\%20de\%20la\%20jungle_12-09-2008.pdf

Craig Louise, Goodson Lisa, Phillimore Jenny and Sankey Sian (2006) Employability Initiatives for Refugees in Europe: Looking at, and Learning from, Good Practice, Report for EQUAL and the Home Office, The Centre for Urban and Regional Studies, The University of Birmingham, Edgbason.

Doraï Kamel (2010) Les Irakiens en Suède : exil et recomposition migratoire depuis la chute du régime de Saddam Hussein en 2003, e-migrinter, 5, pp. 13-23, [en ligne]. URL : http://migrinter.labo.univ-poitiers.fr/e-migrinter2010_05_tout/

Doraï Kamel (2006) Les réfugiés palestiniens du Liban : une géographie de l'exil, Paris, CNRS Editions, 256 p.

Ducheny Marie (2008) Le dispositif national d'accueil et l'accès au logement : enquête sur les relations entre l'État et les réfugiés statutaires, Thèse de doctorat en sociologie, Université Paris 5, 407 p.

Dufoix Stéphane (2000) Fausses évidences. Statut de réfugié et politisation, Revue Européenne de Migrations Internationales, 16 (3), pp. 147-164, [en ligne]. URL : http://www.persee.fr/doc/remi_0765-0752_2000_num_16_3_1745 
Étiemble Angélina (2004) Les Tamouls du Sri Lanka dans la région parisienne. L'emprise du politique, Revue Française des Affaires sociales, 2, pp. 145-164.

Fassin Didier (2010) La raison humanitaire. Une histoire morale du temps présent, Paris, Le Seuil, 368 p.

Ferris Elizabeth G. (Ed.) (1985) Refugees and World Politics, New York, Praeger Publishers Inc, $240 \mathrm{p}$.

Fiddian-Qasmiyeh Elena (2014) Gender and Forced Migration, in Elena FiddianQasmiyeh, Gil Loescher, Katy Long and Nando Sigona Eds., The Handbook of Refugee and Forced Migration Studies, Oxford, Oxford University Press, pp. 395-408.

Fiddian-Qasmiyeh Elena, Loescher Gil, Long Katy and Sigona Nando (Eds.) (2014) The Handbook of Refugee and Forced Migration Studies, Oxford, Oxford University Press, 776 p.

Franguiadakis Spyros, Jaillardon Edith et Belkis Dominique (2004) En quête $d$ 'asile. Aide associative et accès au(x) droit(s), Paris, LGDJ, $287 \mathrm{p}$.

Goodwin-Gill Guy S. (2014) The international Law of Refugee Protection, in Elena Fiddian-Qasmiyeh, Gil Loescher, Katy Long and Nando Sigona Eds., The Handbook of Refugee and Forced Migration Studies, Oxford, Oxford University Press, pp. 36-47.

Grinberg Léon et Grinberg Rebeca (1987) Psychanalyse du migrant et de l'exilé, Lyon, CLE, 289 p.

Hachimi Alaoui Myriam (2007) Les Chemins de l'exil. Les Algériens exilés en France et au Canada depuis les années 1990, Paris, L'Harmattan, 202 p.

Halluin-Mabillot Estelle (d') (2012) Les épreuves de l'asile. Associations et réfugiés face aux politiques du soupçon, Paris, EHESS, $301 \mathrm{p}$.

Harrell-Bond Barbara E. (1986) Imposing Aid: Emergency Assistance to Refugees, Oxford, Oxford University Press, 440 p.

Hathaway James C. (2007) Forced Migration Studies: Could we Agree just to "Date"?, Journal of Refugee Studies, 3 (20), pp. 349-369.

Kobelinsky Carolina (2010) L'accueil des demandeurs d'asile. Une ethnographie de l'attente, Paris, Édition du Cygne, 269 p.

Kogan Irena (2003) Ex-Yugoslavs in the Austrian and Swedish labor markets: The significance of period of migration and the effect of citizenship acquisition, Journal of Ethnic and Migration Studies, 29, pp. 595-622.

Korac Maja (2003) Integration and How We Facilitate It: A comparative Study of the Settlement Experiences of Refugees in Italy and the Netherlands, Sociology, 37 (1), pp. 51-68.

Lassailly-Jacob Véronique (1999) Migrants malgré eux : une proposition de typologie, in Véronique Lassailly-Jacob, Jean-Yves Marchal et André Quesnel Éds., Déplacés et réfugiés : la mobilité sous contrainte, Paris, IRD, pp. 27-48.

Lundborg Per (2010) Refugees' integration: Lagging Wages or Lagging employment?, Working paper, 2, Stockholm, CEIFO, 29 p.

Malkki Liisa H. (1995) Refugees and Exile: From "Refugee Studies" to the National Order of Things, Annual Review of Anthropology, 24, pp. 495-523. 
Martin David A. (1982) Large-Scale Migrations of Asylum Seekers, American Journal of International Law, 76, pp. 598-605.

Masse Jean-Pierre (2001) Genèse et institutionnalisation du dispositif d'accueil des réfugiés politiques en France, Les cahiers de la sécurité intérieure, 45, pp. $45-68$.

Meslin Karine (2011) Les réfugiés cambodgiens, des ouvriers dociles ? Genèse et modes de pérennisation d'un stéréotype en migration, Revue Européenne des Migrations Internationales, 27 (3), pp. 83-101, [en ligne]. URL : https://journals. openedition.org/remi/5646

Migreurop (2017) Atlas des migrants en Europe. Approches critiques des politiques migratoires, Paris, Armand Colin, $176 \mathrm{p}$.

Noiriel Gérard (1998) Réfugiés et sans papiers : la République face au droit $d^{\prime}$ asile, XIXe-XXe siècle, Paris, Hachette Littérature, $355 \mathrm{p}$.

Noiriel Gérard (1997) Représentation nationale et catégories sociales. L'exemple des réfugiés politiques, Genèses, 26, pp. 25-54.

Pestre Élise (2010) La vie psychique des réfugiés, Paris, Payot et Rivages, 377 p.

Rea Andrea and Wets Johan (Eds.) (2015) The Long and Winding Road to Employment. An Analysis of the Labour Market Careers of Asylum Seekers and Refugees in Belgium, Gand, Academia Press, 186 p. [online]. URL: http://www. myria.be/files/Careers_LR.pdf

Saglio-Yatzimirsky Marie-Caroline (2018) La voix de ceux qui crient. Rencontre avec des demandeurs d'asile, Paris, Albin Michel, 318 p.

Spire Alexis (2004) Les réfugiés, une main-d'œuvre à part ? Conditions de séjour et d'emploi, France, 1945-1975, Revue Européenne des Migrations Internationales, 20 (2), pp. 13-38, [en ligne]. URL : https://journals.openedition. org/remi/963

Tcholakova Albena (2016) Le remaniement identitaire entre reconnaissance et maintien de la cohérence biographique. L'exemple des réfugié.e·s en France, Sociologie, 7 (1), pp. 59-76.

Tcholakova Albena (2012) En quête de travail, enjeux de reconnaissance et remaniement identitaire : approche comparée France-Bulgarie de carrières professionnelles de réfugiés, Thèse de doctorat en sociologie et en sciences politiques (cotutelle de thèse avec la Nouvelle Université Bulgare), Université Lumière Lyon 2, $457 \mathrm{p}$.

Turton David (2003) Conceptualising Forced Migration, RSC Working Paper, Refugee Studies Centre, University of Oxford, 2, pp. 1-19.

Turton David (1996) Migrants and Refugees: a Mursi case study, in Allen Tim Ed., In Search of Cool Ground: War, Flight, and Homecoming in Northeast Africa, Trenton, Africa Word Press, pp. 96-110.

United Nation Hight Commissioner for Refugees (UNHCR) (2013a) A new beginning. Refugees Integration in Europe, UNHCR, 146 p.

United Nation Hight Commissioner for Refugees (UNHCR) (2013b) Vers un nouveau départ : I'intégration des réfugiés en France, UNHCR, 92 p. 
Vabre Frédéric (2004) L'engagement comme ressource facilitant l'intégration. Le cas des réfugiés latino-américains en Suède, Revue Européenne des Migrations Internationales, 20 (2), pp. 187-194, [en ligne]. URL : https://journals.openedition. org/remi/990

Vahabi Nader (2008) Sociologie d'une mémoire déchirée. Le cas des exilés iraniens, Paris, L'Harmattan, $246 \mathrm{p}$.

Valluy Jérôme (2009) Rejet des exilés. Le grand retournement du droit de l'asile, Éditions du Croquant, $382 \mathrm{p}$.

Valtonen Kathleen (2001) Social work with immigrants and refugees: developing a particiaption-based framework for anti-opressive practice, British Journal of Social Work, 31, pp. 955-960.

Van Hear Nicholas (2014) Refugees, Diasporas and Transnationalism, in Elena Fiddian-Qasmiyeh, Gil Loescher, Katy Long and Nando Sigona Eds., The Handbook of Refugee and Forced Migration Studies, Oxford, Oxford University Press, pp. 176-187.

Vinatier Laurent (2009) Un modèle de parcours migratoire : Le cas des réfugiés tchétchènes en Europe, EurOrient, 29, pp. 67-90.

Weiss Thomas G. (2013) Humanitarian Business, Cambridge, UK Polity Press, 200 p.

Yi Cheung Sin and Phillimore Jenny (2013) Social networks, social capital and refugee integration, Research Report for Nuffield Foundation, 54 p. 